CUBO A Mathematical Journal Vol.19, $N^{\underline{O}} 01$, (53-7r). March 2017

\title{
Inequalities for Chebyshev Functional in Banach Algebras
}

\author{
S. S. Dragomir ${ }^{1}$, M. V. BoldeA ${ }^{2}$ And M. Megan ${ }^{3}$ \\ 1 Mathematics, School of Engineering \& Science, Victoria University, \\ PO Box 14428, Melbourne City, MC 8001, Australia. \\ School of Computational $\&$ Applied Mathematics, University of the Witwatersrand, . \\ Private Bag 3, Johannesburg 2050, South Africa. \\ 2 Mathematics and Statistics, Banat University of Agricultural Sciences and Veterinary Medicine \\ Timişoara, \\ 119 Calea Aradului, 300645, Timişoara, România \\ 3 Department of Mathematics, West University of Timişoara, \\ B-dul V. Pârvan 4, 1900-Timişoara, România \\ sever.dragomir@vu.edu.au, http://rgmia.org/dragomir
}

\begin{abstract}
By utilizing some identities for double sums, some new inequalities for the Chebyshev functional in Banach algebras are given. Some examples for the exponential and resolvent functions on Banach algebras are also provided.
\end{abstract}

\section{RESUMEN}

Usando algunas identidades para sumas dobles, encontramos algunas nuevas desigualdades para el funcional de Chebyshev en álgebras de Banach. También entregamos algunos ejemplos para las funciones exponencial y resolvente en álgebras de Banach.

Keywords and Phrases: Banach algebras, Power series, Exponential function, Resolvent function, Norm inequalities.

2010 AMS Mathematics Subject Classification: 47A63; 47A99. 


\section{Introduction}

In 1950, M. Biernacki, H. Pidek and C. Ryll-Nardzewski [2] established the following discrete version of Grüss' inequality, see also [39, Ch. X]:

Theorem 1.1. Let $\mathrm{a}=\left(\mathrm{a}_{1}, \ldots, \mathrm{a}_{\mathrm{n}}\right), \mathrm{b}=\left(\mathrm{b}_{1}, \ldots, \mathrm{b}_{\mathrm{n}}\right)$ be two n-tuples of real numbers such that $\mathrm{r} \leq \mathrm{a}_{\mathrm{i}} \leq \mathrm{R}$ and $\mathrm{s} \leq \mathrm{b}_{\mathrm{i}} \leq \mathrm{S}$ for $\mathrm{i}=1, \ldots, \mathrm{n}$. Then one has the inequality:

$$
\begin{aligned}
& \left|\frac{1}{n} \sum_{i=1}^{n} a_{i} b_{i}-\frac{1}{n} \sum_{i=1}^{n} a_{i} \cdot \frac{1}{n} \sum_{i=1}^{n} b_{i}\right| \\
& \leq \frac{1}{n}\left[\frac{n}{2}\right]\left(1-\frac{1}{n}\left[\frac{n}{2}\right]\right)(R-r)(S-s)
\end{aligned}
$$

when $[x]$ is the integer part of $x, x \in \mathbb{R}$.

A weighted version of Grüss' discrete inequality was proved by J.E. Pečarić in 1979, see for instance [39, Ch. X]:

Theorem 1.2. Let $\mathrm{a}, \mathrm{b}$ be two monotonic $\mathrm{n}$-tuples and $\mathrm{p}$ a positive one. Then

$$
\begin{aligned}
& \left|\frac{1}{P_{n}} \sum_{i=1}^{n} p_{i} a_{i} b_{i}-\frac{1}{P_{n}} \sum_{i=1}^{n} p_{i} a_{i} \cdot \frac{1}{P_{n}} \sum_{i=1}^{n} p_{i} b_{i}\right| \\
& \leq\left|a_{n}-a_{1}\right|\left|b_{n}-b_{1}\right| \max _{1 \leq k \leq n-1}\left(\frac{P_{k} \bar{P}_{k+1}}{P_{n}^{2}}\right)
\end{aligned}
$$

where $\mathrm{P}_{\mathrm{n}}:=\sum_{i=1}^{\mathrm{n}} \mathrm{p}_{\mathrm{i}}, \overline{\mathrm{P}}_{\mathrm{k}+1}=\mathrm{P}_{\mathrm{n}}-\mathrm{P}_{\mathrm{k}+1}$.

In 1981, A. Lupaş [39, Ch. X] proved some similar results for the first difference of a as follows :

Theorem 1.3. Let $\mathrm{a}, \mathrm{b}$ two monotonic $\mathrm{n}$-tuples in the same sense and $\mathrm{p}$ a positive $n$-tuple. Then

$$
\begin{aligned}
& \min _{1 \leq i \leq n-1}\left|a_{i+1}-a_{i}\right| \min _{1 \leq i \leq n-1}\left|b_{i+1}-b_{i}\right|\left[\frac{1}{P_{n}} \sum_{i=1}^{n} i^{2} p_{i}-\left(\frac{1}{P_{n}} \sum_{i=1}^{n} i p_{i}\right)^{2}\right] \\
& \leq \frac{1}{P_{n}} \sum_{i=1}^{n} p_{i} a_{i} b_{i}-\frac{1}{P_{n}} \sum_{i=1}^{n} p_{i} a_{i} \cdot \frac{1}{P_{n}} \sum_{i=1}^{n} p_{i} b_{i} \\
& \leq \max _{1 \leq i \leq n-1}\left|a_{i+1}-a_{i}\right| \max _{1 \leq i \leq n-1}\left|b_{i+1}-b_{i}\right|\left[\frac{1}{P_{n}} \sum_{i=1}^{n} i^{2} p_{i}-\left(\frac{1}{P_{n}} \sum_{i=1}^{n} i p_{i}\right)^{2}\right] .
\end{aligned}
$$

If there exists the numbers $\overline{\mathrm{a}}, \overline{\mathrm{a}}_{1}, \mathrm{r}, \mathrm{r}_{1},\left(\mathrm{rr}_{1}>0\right)$ such that $\mathrm{a}_{\mathrm{k}}=\overline{\mathrm{a}}+\mathrm{kr}$ and $\mathrm{b}_{\mathrm{k}}=\overline{\mathrm{a}}_{1}+k \mathrm{r}_{1}$, then in (1.3) the equality holds.

For some generalizations of Gruss' inequality for isotonic linear functionals defined on certain spaces of mappings see Chapter $\mathrm{X}$ of the book [39] where further references are given .

For related results, see [1]-[21], [25]-[31] and [34]-[44]. 


\section{Some Facts on Banach Algebras}

In order to extend the above results for Banach algebras, we need some preliminary facts as follows:

Let $\mathcal{B}$ be an algebra. An algebra norm on $\mathcal{B}$ is a map $\|\cdot\|: \mathcal{B} \rightarrow[0, \infty)$ such that $(\mathcal{B},\|\cdot\|)$ is a normed space, and, further:

$$
\|\mathrm{ab}\| \leq\|\mathrm{a}\|\|\mathrm{b}\|
$$

for any $\mathrm{a}, \mathrm{b} \in \mathcal{B}$. The normed algebra $(\mathcal{B},\|\cdot\|)$ is a Banach algebra if $\|\cdot\|$ is a complete norm.

We assume that the Banach algebra is unital, this means that $\mathcal{B}$ has an identity 1 and that $\|1\|=1$.

Let $\mathcal{B}$ be a unital algebra. An element $a \in \mathcal{B}$ is invertible if there exists an element $b \in \mathcal{B}$ with $a b=b a=1$. The element $b$ is unique; it is called the inverse of $a$ and written $a^{-1}$ or $\frac{1}{a}$. The set of invertible elements of $\mathcal{B}$ is denoted by $\operatorname{Inv} \mathcal{B}$. If $a, b \in \operatorname{Inv} \mathcal{B}$ then $a b \in \operatorname{Inv} \mathcal{B}$ and $(a b)^{-1}=b^{-1} a^{-1}$.

For a unital Banach algebra we also have:

(i) If $a \in \mathcal{B}$ and $\lim _{n \rightarrow \infty}\left\|a^{n}\right\|^{1 / n}<1$, then $1-a \in \operatorname{Inv} \mathcal{B}$;

(ii) $\{a \in \mathcal{B}:\|1-a\|<1\} \subset \operatorname{Inv} \mathcal{B}$;

(iii) $\operatorname{Inv} \mathcal{B}$ is an open subset of $\mathcal{B}$;

(iv) The map $\operatorname{Inv} \mathcal{B} \ni a \longmapsto a^{-1} \in \operatorname{Inv} \mathcal{B}$ is continuous.

For simplicity, we denote $\lambda 1$, where $\lambda \in \mathbb{C}$ and 1 is the identity of $\mathcal{B}$, by $\lambda$. The resolvent set of $a \in \mathcal{B}$ is defined by

$$
\rho(a):=\{\lambda \in \mathbb{C}: \lambda-a \in \operatorname{Inv} \mathcal{B}\}
$$

the spectrum of $a$ is $\sigma(a)$, the complement of $\rho(a)$ in $\mathbb{C}$, and the resolvent function of $a$ is $R_{a}: \rho(a) \rightarrow \operatorname{Inv} \mathcal{B}, R_{a}(\lambda):=(\lambda-a)^{-1}$. For each $\lambda, \gamma \in \rho(a)$ we have the identity

$$
R_{a}(\gamma)-R_{a}(\lambda)=(\lambda-\gamma) R_{a}(\lambda) R_{a}(\gamma)
$$

We also have that $\sigma(a) \subset\{\lambda \in \mathbb{C}:|\lambda| \leq\|\mathbf{a}\|\}$. The spectral radius of $\mathbf{a}$ is defined as $v(\mathbf{a})=$ $\sup \{|\lambda|: \lambda \in \sigma(a)\}$.

If $\mathrm{a}, \mathrm{b}$ are commuting elements in $\mathcal{B}$, i.e. $\mathrm{ab}=\mathrm{b} a$, then

$$
v(a b) \leq v(a) v(b) \text { and } v(a+b) \leq v(a)+v(b)
$$

Let $f$ be an analytic functions on the open disk $D(0, R)$ given by the power series $f(\lambda):=$ $\sum_{j=0}^{\infty} \alpha_{j} \lambda^{j}(|\lambda|<R)$. If $v(a)<R$, then the series $\sum_{j=0}^{\infty} \alpha_{j} a^{j}$ converges in the Banach algebra $\mathcal{B}$ because $\sum_{j=0}^{\infty}\left|\alpha_{j}\right|\left\|a^{j}\right\|<\infty$, and we can define $f(a)$ to be its sum. Clearly $f(a)$ is well defined and 
there are many examples of important functions on a Banach algebra $\mathcal{B}$ that can be constructed in this way. For instance, the exponential map on $\mathcal{B}$ denoted exp and defined as

$$
\exp a:=\sum_{j=0}^{\infty} \frac{1}{j !} a^{j} \text { for each } a \in \mathcal{B} \text {. }
$$

If $\mathcal{B}$ is not commutative, then many of the familiar properties of the exponential function from the scalar case do not hold. The following key formula is valid, however with the additional hypothesis of commutativity for $a$ and $b$ from $\mathcal{B}$

$$
\exp (a+b)=\exp (a) \exp (b)
$$

In a general Banach algebra $\mathcal{B}$ it is difficult to determine the elements in the range of the exponential $\operatorname{map} \exp (\mathcal{B})$, i.e. the element which have a "logarithm". However, it is easy to see that if a is an element in $B$ such that $\|1-a\|<1$, then $a$ is in $\exp (\mathcal{B})$. That follows from the fact that if we set

$$
b=-\sum_{n=1}^{\infty} \frac{1}{n}(1-a)^{n}
$$

then the series converges absolutely and, as in the scalar case, substituting this series into the series expansion for $\exp (b)$ yields $\exp (b)=a$.

It is known that if $x$ and $y$ are commuting, i.e. $x y=y x$, then the exponential function satisfies the property

$$
\exp (x) \exp (y)=\exp (y) \exp (x)=\exp (x+y) \text {. }
$$

Also, if $x$ is invertible and $a, b \in \mathbb{R}$ with $a<b$ then

$$
\int_{a}^{b} \exp (t x) d t=x^{-1}[\exp (b x)-\exp (a x)] .
$$

Moreover, if $x$ and $y$ are commuting and $y-x$ is invertible, then

$$
\begin{aligned}
\int_{0}^{1} \exp ((1-s) x+s y) d s & =\int_{0}^{1} \exp (s(y-x)) \exp (x) d s \\
& =\left(\int_{0}^{1} \exp (s(y-x)) d s\right) \exp (x) \\
& =(y-x)^{-1}[\exp (y-x)-I] \exp (x) \\
& =(y-x)^{-1}[\exp (y)-\exp (x)] .
\end{aligned}
$$

Inequalities for functions of operators in Hilbert spaces may be found in the papers [12], [11] and in the recent monographs [22], [23], [32] and the references therein.

The following inequality of Grüss-Lupaş type in Banach algebras holds: 
Theorem 2.1. Let $\mathcal{B}$ be a Banach algebra over $\mathbb{K}(=\mathbb{R}, \mathbb{C}), a_{i}, b_{i} \in \mathcal{B}$ and $\alpha_{i} \in \mathbb{K}(i=1, \ldots, n)$. Then we have the inequality:

$$
\begin{aligned}
& \left\|\sum_{i=1}^{n} \alpha_{i} \sum_{i=1}^{n} \alpha_{i} a_{i} b_{i}-\sum_{i=1}^{n} \alpha_{i} a_{i} \sum_{i=1}^{n} \alpha_{i} b_{i}\right\| \\
& \leq \max _{1 \leq j \leq n-1}\left\|a_{j+1}-a_{j}\right\| \max _{1 \leq j \leq n-1}\left\|b_{j+1}-b_{j}\right\| \\
& \times\left[\sum_{i=1}^{n}\left|\alpha_{i}\right| \sum_{i=1}^{n} i^{2}\left|\alpha_{i}\right|-\left(\sum_{i=1}^{n} i\left|\alpha_{i}\right|\right)^{2}\right]
\end{aligned}
$$

The inequality (2.1) is sharp in the sense that the multiplicative constant $\mathrm{C}=1$ in the right membership can not be replaced by a smaller one.

Let $\alpha_{n}$ be nonzero complex numbers and let

$$
R:=\frac{1}{\limsup \left|\alpha_{n}\right|^{\frac{1}{n}}}
$$

Clearly $0 \leq \mathrm{R} \leq \infty$, but we consider only the case $0<\mathrm{R} \leq \infty$.

Denote by:

$$
D(0, R)= \begin{cases}\{\lambda \in \mathbb{C}:|\lambda|<R\}, & \text { if } R<\infty \\ \mathbb{C}, & \text { if } R=\infty,\end{cases}
$$

consider the functions:

$$
\lambda \mapsto f(\lambda): D(0, R) \rightarrow \mathbb{C}, f(\lambda):=\sum_{n=0}^{\infty} \alpha_{n} \lambda^{n}
$$

and

$$
\lambda \mapsto f_{A}(\lambda): D(0, R) \rightarrow \mathbb{C}, f_{A}(\lambda):=\sum_{n=0}^{\infty}\left|\alpha_{n}\right| \lambda^{n} .
$$

Let $\mathcal{B}$ be a unital Banach algebra and 1 its unity. Denote by

$$
B(0, R)= \begin{cases}\{x \in \mathcal{B}:\|x\|<R\}, & \text { if } R<\infty \\ \mathcal{B}, & \text { if } R=\infty\end{cases}
$$

We associate to $f$ the map:

$$
x \mapsto \widetilde{\mathrm{f}}(x): B(0, R) \rightarrow \mathcal{B}, \widetilde{\mathrm{f}}(x):=\sum_{n=0}^{\infty} \alpha_{n} x^{n}
$$


Obviously, $\tilde{f}$ is correctly defined because the series $\sum_{n=0}^{\infty} \alpha_{n} x^{n}$ is absolutely convergent, since $\sum_{n=0}^{\infty}\left\|\alpha_{n} x^{n}\right\| \leq \sum_{n=0}^{\infty}\left|\alpha_{n}\right|\|x\|^{n}$.

Making use of Theorem 2.1 we have the following inequality for power series:

Theorem 2.2. Let $\mathrm{f}(\lambda)=\sum_{n=0}^{\infty} \alpha_{n} \lambda^{n}$ be a power series that is convergent on the open disk $\mathrm{D}(0, \mathrm{R})$, with $\mathrm{R}>0$. If $x, y \in \mathcal{B}$ with $x y=y x$ and $\|x\|,\|y\| \leq 1$, then we have for $\lambda \in \mathbb{C}$ with $|\lambda|<\mathrm{R}$ the inequality:

$$
\begin{aligned}
& \|\tilde{f}(\lambda \cdot 1) \tilde{f}(\lambda x y)-\tilde{f}(\lambda x) \tilde{f}(\lambda y)\| \\
& \leq\|x-1\|\|y-1\|\left\{f_{A}(|\lambda|)\left[|\lambda| f_{A}^{\prime}(|\lambda|)+|\lambda|^{2} f_{A}^{\prime \prime}(|\lambda|)\right]-\left[|\lambda| f_{A}^{\prime}(|\lambda|)\right]^{2}\right\} .
\end{aligned}
$$

Motivated by the above results we establish in this paper other similar inequalities for the norm of the Chebyshev difference

$$
\tilde{f}(\lambda \cdot 1) \tilde{f}(\lambda x y)-\tilde{f}(\lambda x) \tilde{f}(\lambda y)
$$

by the use of some discrete inequalities of Grüss' type.

First we establish some identities of interest in Banach algebras,

\section{Identities in Banach Algebras}

Consider the Chebyshev functional defined for $\mathbf{p}=\left(\mathrm{p}_{1}, \ldots, \mathrm{p}_{\mathrm{n}}\right) \in \mathbb{K}^{\mathrm{n}}, \mathbf{a}=\left(\mathrm{a}_{1}, \ldots, \mathrm{a}_{\mathrm{n}}\right) \in \mathcal{B}^{\mathrm{n}}$ and $\mathbf{b}=\left(b_{1}, \ldots, b_{n}\right) \in \mathcal{B}^{n}$, where $\mathcal{B}$ is a Banach algebra over the real or complex number field $\mathbb{K}$ :

$$
T_{n}(\mathbf{p} ; \mathbf{a}, \mathbf{b}):=P_{n} \sum_{i=1}^{n} p_{i} a_{i} b_{i}-\sum_{i=1}^{n} p_{i} a_{i} \sum_{i=1}^{n} p_{i} b_{i}
$$

where $P_{n}:=\sum_{i=1}^{n} p_{i}$.

The following particular identities for unweighted means hold as well, where $T_{n}(\mathbf{a}, \mathbf{x})$ is defined as follows:

$$
T_{n}(\mathbf{a}, \mathbf{b}):=\frac{1}{n} \sum_{i=1}^{n} \alpha_{i} b_{i}-\frac{1}{n} \sum_{i=1}^{n} a_{i} \frac{1}{n} \sum_{i=1}^{n} b_{i}
$$

If $\alpha, \beta$ are scalars and $x, y$ are vectors in a Banach algebra $\mathcal{B}$, we denote by

$$
\operatorname{det}\left(\begin{array}{ll}
\alpha & \beta \\
x & y
\end{array}\right):=\alpha y-\beta x \in \mathcal{B}
$$

The first result is embodied in the following 
Theorem 3.1. Let $\mathbf{p}=\left(\mathrm{p}_{1}, \ldots, \mathrm{p}_{\mathrm{n}}\right) \in \mathbb{K}^{\mathrm{n}}$, and $\boldsymbol{a}=\left(\mathrm{a}_{1}, \ldots, \mathrm{a}_{\mathrm{n}}\right), \boldsymbol{b}=\left(\mathrm{b}_{1}, \ldots, \mathrm{b}_{\mathfrak{n}}\right) \in \mathcal{B}^{\mathrm{n}}$. If we define

$$
\begin{aligned}
P_{i} & :=\sum_{k=1}^{i} p_{k}, \bar{P}_{i}:=P_{n}-P_{i}, i \in\{1, \ldots, n-1\}, \\
A_{i}(\mathbf{p}): & =\sum_{k=1}^{i} p_{k} a_{k}, \bar{A}_{i}(\mathbf{p}):=A_{n}(\mathbf{p})-A_{i}(\mathbf{p}), i \in\{1, \ldots, n-1\},
\end{aligned}
$$

then we have the identity

$$
\begin{aligned}
\mathrm{T}_{\mathrm{n}}(\mathbf{p} ; \mathbf{a}, \mathbf{b}) & =\sum_{i=1}^{n-1} \operatorname{det}\left(\begin{array}{cc}
\mathrm{P}_{\mathrm{i}} & \mathrm{P}_{\mathrm{n}} \\
A_{i}(\mathbf{p}) & A_{n}(\mathbf{p})
\end{array}\right) \Delta b_{i} \\
& =\mathrm{P}_{\mathrm{n}} \sum_{i=1}^{n-1} P_{i}\left(\frac{A_{n}(\mathbf{p})}{P_{n}}-\frac{A_{i}(\mathbf{p})}{P_{i}}\right) \Delta b_{i} \\
& \left(\text { if } P_{i} \neq 0, i \in\{1, \ldots, n\}\right) \\
& =\sum_{i=1}^{n-1} P_{i} \bar{P}_{i}\left(\frac{\bar{A}_{i}(\mathbf{p})}{\bar{P}_{i}}-\frac{A_{i}(\mathbf{p})}{P_{i}}\right) \Delta b_{i} \\
& \left(\text { if } P_{i}, \bar{P}_{i} \neq 0, i \in\{1, \ldots, n-1\}\right) ;
\end{aligned}
$$

where $\Delta b_{i}:=b_{i+1}-b_{i}(i \in\{1, \ldots, n-1\})$ is the forward difference.

Proof. We use the following well known summation by parts formula

$$
\sum_{l=p}^{q-1} d_{l} \Delta v_{l}=\left.d_{l} v_{l}\right|_{p} ^{q}-\sum_{l=p}^{q-1} \Delta d_{l} v_{l+1}
$$

where $d_{l}$ and $v_{l}$ are vectors in a linear space, $l=p, \ldots, q(q>p ; p, q$ are natural numbers).

If we choose in (3.4), $p=1, q=n, d_{i}=P_{i} A_{n}(\mathbf{p})-P_{n} A_{i}(\mathbf{p})$ and $v_{i}=b_{i}(i \in\{1, \ldots, n-1\})$, 
then we get

$$
\begin{aligned}
& \sum_{i=1}^{n-1}\left(P_{i} A_{n}(\mathbf{p})-P_{n} A_{i}(\mathbf{p})\right) \Delta b_{i} \\
& =\left.\left[P_{i} A_{n}(\mathbf{p})-P_{n} A_{i}(\mathbf{p})\right] b_{i}\right|_{1} ^{n}-\sum_{i=1}^{n-1} \Delta\left(P_{i} A_{n}(\mathbf{p})-P_{n} A_{i}(\mathbf{p})\right) b_{i+1} \\
& =\left[P_{n} A_{n}(\mathbf{p})-P_{n} A_{n}(\mathbf{p})\right] b_{n}-\left[P_{1} A_{n}(\mathbf{p})-P_{n} A_{1}(\mathbf{p})\right] b_{1} \\
& -\sum_{i=1}^{n-1}\left[P_{i+1} A_{n}(\mathbf{p})-P_{n} A_{i+1}(\mathbf{p})-P_{i} A_{n}(\mathbf{p})+P_{n} A_{i}(\mathbf{p})\right] b_{i+1} \\
& =P_{n} p_{1} a_{1} b_{1}-p_{1} A_{n}(\mathbf{p}) b_{1}-\sum_{i=1}^{n-1}\left(p_{i+1} A_{n}(\mathbf{p})-P_{n} p_{i+1} a_{i+1}\right) b_{i+1} \\
& =P_{n} p_{1} a_{1} b_{1}-p_{1} A_{n}(\mathbf{p}) b_{1}-A_{n}(\mathbf{p}) \sum_{i=1}^{n-1} p_{i+1} b_{i+1}+P_{n} \sum_{i=1}^{n} p_{i+1} a_{i+1} b_{i+1} \\
& =P_{n} \sum_{i=1}^{n} p_{i} a_{i} b_{i}-\sum_{i=1}^{n} p_{i} a_{i} \sum_{i=1}^{n} p_{i} b_{i} \\
& =T_{n}\left(\mathbf{p} ; a_{,} b\right),
\end{aligned}
$$

which produce the first identity in (3.3) .

The second and the third identities are obvious and we omit the details.

Before we prove the second result, we need the following lemma providing an identity that is interesting in itself as well.

Lemma 3.1. Let $\mathbf{p}=\left(\mathrm{p}_{1}, \ldots, \mathrm{p}_{\mathrm{n}}\right) \in \mathbb{K}^{\mathrm{n}}$ and $\boldsymbol{a}=\left(\mathrm{a}_{1}, \ldots, \mathrm{a}_{\mathrm{n}}\right) \in \mathcal{B}^{\mathrm{n}}$. Then we have the equality

$$
\operatorname{det}\left(\begin{array}{cc}
P_{i} & P_{n} \\
A_{i}(\mathbf{p}) & A_{n}(\mathbf{p})
\end{array}\right)=\sum_{j=1}^{n-1} P_{\min \{i, j\}} \bar{P}_{\max \{i, j\}} \Delta a_{j},
$$

for each $i \in\{1, \ldots, n-1\}$.

Proof. Define, for $i \in\{1, \ldots, n-1\}$,

$$
K(i):=\sum_{j=1}^{n-1} P_{\min \{i, j\}} \bar{P}_{\max \{i, j\}} \Delta a_{j} .
$$


We have

$$
\begin{aligned}
K(i) & =\sum_{j=1}^{i} P_{\min \{i, j\}} \bar{P}_{\max \{i, j\}} \Delta a_{j}+\sum_{j=i+1}^{n-1} P_{\min \{i, j\}} \bar{P}_{\max \{i, j\}} \Delta a_{j} \\
& =\sum_{j=1}^{i} P_{j} \bar{P}_{i} \Delta a_{j}+\sum_{j=i+1}^{n-1} P_{i} \bar{P}_{j} \Delta a_{j} \\
& =\bar{P}_{i} \sum_{j=1}^{i} P_{j} \Delta a_{j}+P_{i} \sum_{j=i+1}^{n-1} \bar{P}_{j} \Delta a_{j} .
\end{aligned}
$$

Using the summation by parts formula, we have

$$
\begin{aligned}
\sum_{j=1}^{i} P_{j} \Delta a_{j} & =\left.P_{j} a_{j}\right|_{1} ^{i+1}-\sum_{j=1}^{i}\left(P_{j+1}-P_{j}\right) a_{j+1} \\
& =P_{i+1} a_{i+1}-p_{1} a_{1}-\sum_{j=1}^{i} p_{j+1} a_{j+1} \\
& =P_{i+1} a_{i+1}-\sum_{j=1}^{i+1} p_{j} a_{j}
\end{aligned}
$$

and

$$
\begin{aligned}
\sum_{j=i+1}^{n-1} \bar{P}_{j} \Delta a_{j} & =\left.\bar{P}_{j} a_{j}\right|_{i+1} ^{n}-\sum_{j=i+1}^{n-1}\left(\bar{P}_{j+1}-\bar{P}_{j}\right) a_{j+1} \\
& =\bar{P}_{n} a_{n}-\bar{P}_{i+1} a_{i+1}-\sum_{j=i+1}^{n-1}\left(P_{n}-P_{j+1}-P_{n}+P_{j}\right) a_{j+1} \\
& =-\bar{P}_{i+1} a_{i+1}+\sum_{j=i+1}^{n-1} p_{j+1} a_{j+1} .
\end{aligned}
$$

Using (3.7) and (3.8) we have

$$
\begin{aligned}
K(i) & =\bar{P}_{i}\left(P_{i+1} a_{i+1}-\sum_{j=1}^{i+1} p_{j} a_{j}\right)+P_{i}\left(\sum_{j=i+1}^{n-1} p_{j+1} a_{j+1}-\bar{P}_{i+1} a_{i+1}\right) \\
& =\bar{P}_{i} P_{i+1} a_{i+1}-P_{i} \bar{P}_{i+1} a_{i+1}-\bar{P}_{i} \sum_{j=1}^{i+1} p_{j} a_{j}+P_{i} \sum_{j=i+1}^{n-1} p_{j+1} a_{j+1} \\
& =\left[\left(P_{n}-P_{i}\right) P_{i+1}-P_{i}\left(P_{n}-P_{i+1}\right)\right] a_{i+1} \\
& +P_{i} \sum_{j=i+1}^{n-1} p_{j+1} a_{j+1}-\bar{P}_{i} \sum_{j=1}^{i+1} p_{j} a_{j}
\end{aligned}
$$




$$
\begin{aligned}
& =P_{n} p_{i+1} a_{i+1}+P_{i} \sum_{j=i+1}^{n-1} p_{j+1} a_{j+1}-\bar{P}_{i} \sum_{j=1}^{i+1} p_{j} a_{j} \\
& =\left(P_{i}+\bar{P}_{i}\right) p_{i+1} a_{i+1}+P_{i} \sum_{j=i+1}^{n-1} p_{j+1} a_{j+1}-\bar{P}_{i} \sum_{j=1}^{i+1} p_{j} a_{j} \\
& =P_{i} \sum_{j=i+1}^{n-1} p_{j} a_{j}-\bar{P}_{i} \sum_{j=1}^{i} p_{j} a_{j}=P_{i} \bar{A}_{i}(\mathbf{p})-\bar{P}_{i} A_{i}(\mathbf{p}) \\
& =\operatorname{det}\left(\begin{array}{cc}
P_{i} & P_{n} \\
A_{i}(\mathbf{p}) & A_{n}(\mathbf{p})
\end{array}\right) ;
\end{aligned}
$$

and the identity is proved.

We are able now to state and prove the second identity for the Čebyšev functional

Theorem 3.2. With the assumptions of Theorem 3.1, we have the equality

$$
\mathrm{T}_{\mathrm{n}}(\mathbf{p} ; \mathbf{a}, \mathbf{b})=\sum_{i=1}^{\mathrm{n}-1} \sum_{j=1}^{\mathrm{n}-1} \mathrm{P}_{\min \{i, j\}} \overline{\mathrm{P}}_{\max \{i, j\}} \Delta \mathrm{a}_{j} \Delta \mathrm{b}_{i} .
$$

The proof is obvious by Theorem 3.1 and Lemma 3.1 .

\section{Some New Inequalities}

The following result holds

Theorem 4.1. Let $\mathbf{p}=\left(\mathrm{p}_{1}, \ldots, \mathrm{p}_{\mathrm{n}}\right) \in \mathbb{K}^{\mathrm{n}}$, and $\boldsymbol{a}=\left(\mathrm{a}_{1}, \ldots, \mathrm{a}_{\mathrm{n}}\right), \boldsymbol{b}=\left(\mathrm{b}_{1}, \ldots, \mathrm{b}_{\mathrm{n}}\right) \in \mathcal{B}^{\mathrm{n}}$. Then we have the inequalities

$$
\leq\left\{\begin{array}{l}
\left\|T_{n}(\mathbf{p} ; \mathbf{a}, \mathbf{b})\right\| \\
\max _{1 \leq i \leq n-1}\left\|\operatorname{det}\left(\begin{array}{cc}
P_{i} & P_{n} \\
A_{i}(\mathbf{p}) & A_{n}(\mathbf{p})
\end{array}\right)\right\| \sum_{i=1}^{n-1}\left\|\Delta b_{i}\right\| ; \\
\text { for } p>1, \frac{1}{p}+\frac{1}{q}=1 ; \\
\left.\sum_{i=1}^{n-1}\left\|\operatorname{det}\left(\begin{array}{cc}
P_{i} & P_{n} \\
A_{i}(\mathbf{p}) & A_{n}(\mathbf{p})
\end{array}\right)\right\|^{q}\right)^{1 / q}\left(\sum_{i=1}^{n-1}\left\|\Delta b_{i}\right\|^{p}\right)^{1 / p} \\
\sum_{i=1}^{n-1}\left\|\operatorname{det}\left(\begin{array}{cc}
P_{i} & P_{n} \\
A_{i}(\mathbf{p}) & A_{n}(\mathbf{p})
\end{array}\right)\right\| \max _{1 \leq i \leq n-1}\left\|\Delta b_{i}\right\| .
\end{array}\right.
$$


All the inequalities in (4.1) are sharp in the sense that the constants 1 cannot be replaced by smaller constants.

Proof. Using the first identity in (3.3), we have

$$
\left\|T_{n}(\mathbf{p} ; \mathbf{a}, \mathbf{b})\right\| \leq \sum_{i=1}^{n-1}\left\|\operatorname{det}\left(\begin{array}{cc}
P_{i} & P_{n} \\
A_{i}(\mathbf{p}) & A_{n}(\mathbf{p})
\end{array}\right)\right\|\left\|\Delta b_{i}\right\|
$$

Using Hölder's inequality, we deduce the desired result (4.1) .

Let prove, for instance, that the constant 1 in the second inequality is best possible.

Assume, for $\mathrm{C}>0$, we have that

$$
\left\|T_{n}(\mathbf{p} ; \mathbf{a}, \mathbf{b})\right\| \leq C\left(\sum_{i=1}^{n-1}\left\|\operatorname{det}\left(\begin{array}{cc}
P_{i} & P_{n} \\
A_{i}(\mathbf{p}) & A_{n}(\mathbf{p})
\end{array}\right)\right\|^{q}\right)^{1 / q}\left(\sum_{i=1}^{n-1}\left\|\Delta b_{i}\right\|^{p}\right)^{1 / p}
$$

for $p>1, \frac{1}{p}+\frac{1}{q}=1, n \geq 2$.

If we choose $n=2$, then we get

$$
\mathrm{T}_{2}(\mathbf{p} ; \mathbf{a}, \mathbf{b})=\mathrm{p}_{1} \mathrm{p}_{2}\left(\mathrm{a}_{2}-\mathrm{a}_{1}\right)\left(\mathrm{b}_{2}-\mathrm{b}_{1}\right) .
$$

Also, for $\mathrm{n}=2$,

$$
\left(\sum_{i=1}^{n-1}\left\|\operatorname{det}\left(\begin{array}{cc}
P_{i} & P_{n} \\
A_{i}(\mathbf{p}) & A_{n}(\mathbf{p})
\end{array}\right)\right\|^{q}\right)^{1 / q}=\left|p_{1} p_{2}\right|\left\|a_{2}-a_{1}\right\|
$$

and

$$
\left(\sum_{j=1}^{n-1}\left\|\Delta b_{j}\right\|^{p}\right)^{1 / p}=\left\|b_{2}-b_{1}\right\|
$$

Then by (4.2), holding for $n=2, p_{1}, p_{2} \neq 0, a_{1} \neq a_{2}, b_{2} \neq b_{1}$, we deduce $C \geq 1$, proving that 1 is the best possible constant in that inequality.

The following corollary for the uniform distribution of the probability $\mathbf{p}$ holds. 
Corolary 1. With the assumptions of Theorem 4.1 for $\mathbf{a}$ and $\boldsymbol{b}$, we have the inequalities

$$
\begin{aligned}
& \left\|T_{n}(\mathbf{a}, \mathbf{b})\right\| \\
& \leq \frac{1}{n^{2}} \times\left\{\begin{array}{l}
\max _{1 \leq i \leq n-1}\left\|\operatorname{det}\left(\begin{array}{cc}
i & n \\
\sum_{k=1}^{i} a_{k} & \sum_{k=1}^{n} a_{k}
\end{array}\right)\right\| \sum_{i=1}^{n-1}\left\|\Delta b_{i}\right\| ; \\
\left(\sum_{i=1}^{n-1}\left\|\operatorname{det}\left(\begin{array}{cc}
i & n \\
\sum_{k=1}^{i} a_{k} & \sum_{k=1}^{n} a_{k}
\end{array}\right)\right\|^{q}\right)^{1 / q} \\
\text { for } p>1, \frac{1}{p}+\frac{1}{q}=1 ; \\
\left.\sum_{i=1}^{n-1}\left\|\Delta b_{i}\right\|^{p}\right)^{1 / p} \\
\operatorname{det}\left(\begin{array}{cc}
i & n
\end{array}\right)\left\|\max _{1 \leq i \leq n-1}^{i}\right\| \Delta b_{i} \| .
\end{array}\right.
\end{aligned}
$$

The following result may be stated as well.

Theorem 4.2. With the assumptions of Theorem 4.1 and if $\mathrm{P}_{\mathrm{i}} \neq 0(\mathrm{i}=1, \ldots, \mathrm{n})$, then we have the inequalities

$$
\begin{aligned}
& \left\|T_{n}(\mathbf{p} ; \mathbf{a}, \mathbf{b})\right\| \\
& \leq\left|P_{n}\right| \times\left\{\begin{array}{l}
\max _{1 \leq i \leq n-1}\left\|\frac{A_{n}(\mathbf{p})}{P_{n}}-\frac{A_{i}(\mathbf{p})}{P i}\right\| \sum_{i=1}^{n-1}\left|P_{i}\right|\left\|\Delta b_{i}\right\| ; \\
\left(\sum_{i=1}^{n-1}\left|P_{i}\right|\left\|\frac{A_{n}(\mathbf{p})}{P_{n}}-\frac{A_{i}(\mathbf{p})}{P i}\right\|^{q}\right)^{1 / q}\left(\sum_{i=1}^{n-1}\left|P_{i}\right|\left\|\Delta b_{i}\right\|^{p}\right)^{1 / p} \\
\text { for } p>1, \frac{1}{p}+\frac{1}{q}=1 ; \\
\sum_{i=1}^{n-1}\left|P_{i}\right|\left\|\frac{A_{n}(\mathbf{p})}{P_{n}}-\frac{A_{i}(\mathbf{p})}{P i}\right\| \max _{1 \leq i \leq n-1}\left\|\Delta b_{i}\right\| .
\end{array}\right.
\end{aligned}
$$

All the inequalities in (4.4) are sharp in the sense that the constant 1 cannot be replaced by a smaller constant.

Proof. Follows by the second identity in (3.3) and taking into account that

$$
\left\|T_{n}(\mathbf{p} ; \mathbf{a}, \mathbf{b})\right\| \leq\left|P_{n}\right| \sum_{i=1}^{n-1}\left\|\frac{A_{n}(\mathbf{p})}{P_{n}}-\frac{A_{i}(\mathbf{p})}{P_{i}}\right\|\left|P_{i}\right|\left\|\Delta b_{i}\right\| .
$$

Using Hölder's weighted inequality, we easily deduce (4.4) .

The sharpness of the constant may be shown in a similar manner. We omit the details. 
The following corollary containing the unweighted inequalities holds.

Corolary 2. With the above assumptions for $\mathbf{a}$ and $\mathbf{b}$ one, has

$$
\begin{aligned}
& \left\|T_{n}(\mathbf{a}, \mathbf{b})\right\| \\
& \left\{\begin{array}{l}
\max _{1 \leq i \leq n-1}\left\|\frac{1}{n} \sum_{k=1}^{n} a_{k}-\frac{1}{i} \sum_{k=1}^{i} a_{k}\right\| \sum_{i=1}^{n-1} i\left\|\Delta b_{i}\right\| ; \\
\left(\sum_{i=1}^{n-1} i\left\|\frac{1}{n} \sum_{k=1}^{n} a_{k}-\frac{1}{i} \sum_{k=1}^{i} a_{k}\right\|^{q}\right)^{1 / q}\left(\sum_{i=1}^{n-1} i\left\|\Delta b_{i}\right\|^{p}\right)^{1 / p} \\
\text { for } p>1, \frac{1}{p}+\frac{1}{q}=1 ; \\
\sum_{i=1}^{n-1} i\left\|\frac{1}{n} \sum_{k=1}^{n} a_{k}-\frac{1}{i} \sum_{k=1}^{i} a_{k}\right\| \max _{1 \leq i \leq n-1}\left\|\Delta b_{i}\right\| .
\end{array}\right.
\end{aligned}
$$

The inequalities in (4.5) are sharp in the sense mentioned above.

Another type of inequalities may be stated if one uses the third identity in (3.3).

Theorem 4.3. With the assumptions in Theorem 4.1 and if $\mathrm{P}_{\boldsymbol{i}}, \overline{\mathrm{P}}_{\mathbf{i}} \neq 0, \mathrm{i} \in\{1, \ldots, \mathrm{n}-1\}$, then we have the inequalities

$$
\leq\left\{\begin{array}{l}
\left\|T_{n}(\mathbf{p} ; \mathbf{a}, \mathbf{b})\right\| \\
\left(\sum_{i=1}^{n-1}\left|P_{i}\right|\left|\bar{P}_{i}\right|\left\|\frac{\bar{A}_{i}(\mathbf{p})}{\bar{P}_{i}}-\frac{A_{i}(\mathbf{p})}{P i}\right\|^{q}\right)^{1 / q}\left(\sum_{i=1}^{n-1}\left|P_{i}\right|\left|\bar{P}_{i}\right|\left\|\Delta b_{i}\right\|^{p}\right)^{1 / p} \\
\text { for } p>1, \frac{1}{p}+\frac{1}{q}=1 ; \\
\sum_{i=1}^{n-1}\left|P_{i}\right|\left|\bar{P}_{i}\right|\left\|\frac{\bar{A}_{i}(\mathbf{p})}{\bar{P}_{i}}-\frac{A_{i}(\mathbf{p})}{P i}\right\| \sum_{i=1}^{n-1}\left|P_{i}\right|\left|\bar{P}_{i}\right|\left\|\Delta b_{i}\right\| ; \\
\bar{P}_{i}-\frac{A_{i}(\mathbf{p})}{P i}\left\|\max _{1 \leq i \leq n-1}\right\| \Delta b_{i} \| .
\end{array}\right.
$$


In particular, if $\mathrm{p}_{\boldsymbol{i}}=\frac{1}{\mathrm{n}}, \boldsymbol{i} \in\{1, \ldots, \mathrm{n}\}$, then we have

$$
\begin{aligned}
& \left\|T_{n}(\mathbf{a}, \mathbf{b})\right\| \\
& \left\{\begin{array}{l}
\max _{1 \leq i \leq n-1}\left\|\frac{1}{n-i} \sum_{k=i+1}^{n} a_{k}-\frac{1}{i} \sum_{k=1}^{i} a_{k}\right\| \\
\times \sum_{i=1}^{n-1} i(n-i)\left\|\Delta b_{i}\right\| ; \\
\left(\sum_{i=1}^{n-1} i(n-i)\left\|\frac{1}{n-i} \sum_{k=i+1}^{n} a_{k}-\frac{1}{i} \sum_{k=1}^{i} a_{k}\right\|^{q}\right)^{1 / q} \\
\times\left(\sum_{i=1}^{n-1} i(n-i)\left\|\Delta b_{i}\right\|^{p}\right)^{1 / p} \text { for } p>1, \frac{1}{p}+\frac{1}{q}=1 ; \\
\sum_{i=1}^{n-1} i(n-i)\left\|\frac{1}{n-i} \sum_{k=i+1}^{n} a_{k}-\frac{1}{i} \sum_{k=1}^{i} a_{k}\right\| \\
\times \max _{1 \leq i \leq n-1}\left\|\Delta b_{i}\right\| .
\end{array}\right.
\end{aligned}
$$

The inequalities in (4.6) and (4.7) are sharp in the above mentioned sense.

A different approach may be considered if one uses the representation in terms of double sums for the Chebyshev functional provided by the Theorem 3.2.

The following result holds.

Theorem 4.4. With the assumptions in Theorem 4.1, we have the inequalities

$$
\leq\left\{\begin{array}{l}
\left\|T_{n}(\mathbf{p} ; \mathbf{a}, \mathbf{b})\right\| \\
\max _{1 \leq i, j \leq n-1}\left\{\left|P_{\min \{i, j\}}\right|\left|\bar{P}_{\max \{i, j\}}\right|\right\} \sum_{i=1}^{n-1}\left\|\Delta a_{i}\right\| \sum_{i=1}^{n-1}\left\|\Delta b_{i}\right\| ; \\
\left.\times\left(\sum_{i=1}^{n-1} \sum_{j=1}^{n-1}\left|P_{\min \{i, j\}}\right|^{q}\left|\bar{P}_{\max \{i, j\}}\right|^{q}\right)^{1 / q}\left\|\Delta a_{i}\right\|^{p}\right)^{1 / p}\left(\sum_{i=1}^{n-1}\left\|\Delta b_{i}\right\|^{p}\right)^{1 / p} \text { for } p>1, \frac{1}{p}+\frac{1}{q}=1 ; \\
\sum_{i=1}^{n-1} \sum_{j=1}^{n-1}\left|P_{\min \{i, j\}}\right|\left|\bar{P}_{\max \{i, j\}}\right| \\
\times \max _{1 \leq i \leq n-1}\left\|\Delta a_{i}\right\| \max _{1 \leq i \leq n-1}\left\|\Delta b_{i}\right\| .
\end{array}\right.
$$

The inequalities are sharp in the sense mentioned above.

The proof follows by the identity (3.9) on using Hölder's inequality for double sums and we omit the details.

The above theorem has some important consequences as follows: 
Corolary 3. Let $\mathbf{p}=\left(\mathrm{p}_{1}, \ldots, \mathrm{p}_{\mathrm{n}}\right) \in \mathbb{K}^{\mathrm{n}}$, and $\boldsymbol{a}=\left(\mathrm{a}_{1}, \ldots, \mathrm{a}_{\mathrm{n}}\right), \boldsymbol{b}=\left(\mathrm{b}_{1}, \ldots, \mathrm{b}_{\mathrm{n}}\right) \in \mathcal{B}^{\mathrm{n}}$. Then we have the inequality

$$
\left\|T_{n}(\mathbf{p} ; \mathbf{a}, \mathbf{b})\right\| \leq \frac{1}{4}\left(\sum_{k=1}^{n}\left|p_{k}\right|\right)^{2} \sum_{i=1}^{n-1}\left\|\Delta a_{i}\right\| \sum_{i=1}^{n-1}\left\|\Delta b_{i}\right\| .
$$

The constant $\frac{1}{4}$ is best possible in (4.9).

Proof. We observe that

$$
\left|P_{\min \{i, j\}}\right|=\left|\sum_{k=1}^{\min \{i, j\}} p_{k}\right| \leq \sum_{k=1}^{\min \{i, j\}}\left|p_{k}\right|
$$

and

for any $1 \leq i, j \leq n-1$.

$$
\left|\overline{\mathrm{P}}_{\max \{i, j\}}\right|=\left|\sum_{k=\max \{i, j\}}^{n} p_{k}\right| \leq \sum_{k=\max \{i, j\}}^{n}\left|p_{k}\right|
$$

This implies that

$$
\begin{aligned}
\left|P_{\min \{i, j\}}\right|\left|\bar{P}_{\max \{i, j\}}\right| & \leq \sum_{k=1}^{\min \{i, j\}}\left|p_{k}\right| \sum_{k=\max \{i, j\}}^{n}\left|p_{k}\right| \\
& \leq \frac{1}{4}\left(\sum_{k=1}^{\min \{i, j\}}\left|p_{k}\right|+\sum_{k=\max \{i, j\}}^{n}\left|p_{k}\right|\right)^{2} \\
& \leq \frac{1}{4}\left(\sum_{k=1}^{n}\left|p_{k}\right|\right)^{2}
\end{aligned}
$$

for any $1 \leq i, j \leq n-1$.

Therefore

$$
\max _{1 \leq i, j \leq n-1}\left\{\left|P_{\min \{i, j\}}\right|\left|\bar{P}_{\max \{i, j\}}\right|\right\} \leq \frac{1}{4}\left(\sum_{k=1}^{n}\left|p_{k}\right|\right)^{2}
$$

and by the first inequality in (4.8) we get (4.9).

To prove the sharpness of the constant $\frac{1}{4}$, assume that (4.9) holds with a constant $C>0$, i.e.

$$
\left\|T_{n}(\mathbf{p} ; \mathbf{a}, \mathbf{b})\right\| \leq \mathrm{C}\left(\sum_{k=1}^{n}\left|p_{k}\right|\right)^{2} \sum_{i=1}^{n-1}\left\|\Delta a_{i}\right\| \sum_{i=1}^{n-1}\left\|\Delta b_{i}\right\| .
$$

If we take in (4.10) $n=2$, then we have

$$
\left\|p_{1} p_{2}\left(a_{2}-a_{1}\right)\left(b_{2}-b_{1}\right)\right\| \leq C\left(\left|p_{1}\right|+\left|p_{2}\right|\right)^{2}\left\|a_{2}-a_{1}\right\|\left\|b_{2}-b_{1}\right\| .
$$

If we take $p_{1}=p_{2}=\frac{1}{2}, a_{2}-a_{1}=\alpha \cdot 1, b_{2}-b_{1}=\beta \cdot 1$ with $\alpha, \beta \neq 0$ then we get $C \geq \frac{1}{4}$, and the proof is completed. 
We have:

Corolary 4. Let $\mathbf{p}=\left(\mathrm{p}_{1}, \ldots, \mathrm{p}_{\mathrm{n}}\right) \in \mathbb{K}^{\mathrm{n}}$, and $\boldsymbol{a}=\left(\mathrm{a}_{1}, \ldots, \mathrm{a}_{\mathrm{n}}\right), \boldsymbol{b}=\left(\mathrm{b}_{1}, \ldots, \mathrm{b}_{\mathrm{n}}\right) \in \mathcal{B}^{\mathrm{n}}$. Then we have the inequality

$$
\begin{aligned}
\left\|T_{n}(\mathbf{p} ; \mathbf{a}, \mathbf{b})\right\| & \leq \frac{1}{4 \mathrm{p}}(\mathrm{n}+1)^{2 p}\left[\sum_{i=1}^{n}\left|p_{i}\right|^{q} \sum_{i=1}^{n} i^{2}\left|p_{i}\right|^{q}-\left(\sum_{i=1}^{n} i\left|p_{i}\right|^{q}\right)^{2}\right]^{1 / q} \\
& \times\left(\sum_{i=1}^{n-1}\left\|\Delta a_{i}\right\|^{p}\right)^{1 / p}\left(\sum_{i=1}^{n-1}\left\|\Delta b_{i}\right\|^{p}\right)^{1 / p}
\end{aligned}
$$

for $p>1, \frac{1}{p}+\frac{1}{q}=1$.

Proof. We observe that by Hölder's inequality we have

$$
\left|P_{\min \{i, j\}}\right|^{\mathrm{q}}=\left|\sum_{k=1}^{\min \{i, j\}} p_{k}\right|^{\mathrm{q}} \leq(\min \{i, j\})^{\mathrm{q}-1} \sum_{k=1}^{\min \{i, j\}}\left|p_{k}\right|^{\mathrm{q}}
$$

and

$$
\left|\bar{P}_{\max \{i, j\}}\right|^{q}=\left|\sum_{k=\max \{i, j\}}^{n} p_{k}\right|^{q} \leq(n-\max \{i, j\}+1)^{q-1} \sum_{k=\max \{i, j\}}^{n}\left|p_{k}\right|^{q}
$$

for any $1 \leq i, j \leq n-1$.

Then

$$
\begin{aligned}
& \left|P_{\min \{i, j\}}\right|^{\mathrm{q}}\left|\bar{P}_{\max \{i, j\}}\right|^{\mathrm{q}} \\
& \leq(n-\max \{i, j\}+1)^{\mathrm{q}-1}(\min \{i, j\})^{\mathrm{q}-1} \sum_{k=1}^{\min \{i, j\}}\left|p_{k}\right|^{\mathrm{q}} \sum_{k=\max \{i, j\}}^{n}\left|p_{k}\right|^{\mathrm{q}} \\
& =[(n-\max \{i, j\}+1)(\min \{i, j\})]^{\mathrm{q}-1} \sum_{k=1}^{\min \{i, j\}}\left|p_{k}\right|^{\mathrm{q}} \sum_{k=\max \{i, j\}}^{n}\left|p_{k}\right|^{\mathrm{q}} .
\end{aligned}
$$

Observe that

$$
(n-\max \{i, j\}+1)(\min \{i, j\}) \leq \frac{1}{4}(n-\max \{i, j\}+1+\min \{i, j\})^{2} .
$$

Since for any $1 \leq i, j \leq n-1$ we have

$$
\max \{i, j\}-\min \{i, j\}=|i-j|
$$

then

$$
(n-\max \{i, j\}+1)(\min \{i, j\}) \leq \frac{1}{4}(n+1-|i-j|)^{2} \leq \frac{1}{4}(n+1)^{2}
$$


and by (4.12) we get

$$
\left|P_{\min \{i, j\}}\right|^{\mathrm{q}}\left|\bar{P}_{\max \{i, j\}}\right|^{\mathrm{q}} \leq\left[\frac{1}{4}(n+1)^{2}\right]^{\mathrm{q}-1} \sum_{k=1}^{\min \{i, j\}}\left|p_{k}\right|^{\mathrm{q}} \sum_{k=\max \{i, j\}}^{n}\left|p_{k}\right|^{\mathrm{q}}
$$

or any $1 \leq i, j \leq n-1$.

Making use of the second inequality in (4.8) we have

$$
\begin{aligned}
& \left\|T_{n}(\mathbf{p} ; \mathbf{a}, \mathbf{b})\right\| \\
& \leq\left(\sum_{i=1}^{n-1} \sum_{j=1}^{n-1}\left|P_{\min \{i, j\}}\right|^{q}\left|\bar{P}_{\max \{i, j\}}\right|^{q}\right)^{1 / q} \\
& \times\left(\sum_{i=1}^{n-1}\left\|\Delta a_{i}\right\|^{p}\right)^{1 / p}\left(\sum_{i=1}^{n-1}\left\|\Delta b_{i}\right\|^{p}\right)^{1 / p} \\
& \leq\left(\left[\frac{1}{4}(n+1)^{2}\right]^{q-1} \sum_{i=1}^{n-1} \sum_{j=1}^{n-1}\left(\sum_{k=1}^{\min \{i, j\}}\left|p_{k}\right|^{q} \sum_{k=\max \{i, j\}}^{n}\left|p_{k}\right|^{q}\right)\right)^{1 / q} \\
& \times\left(\sum_{i=1}^{n-1}\left\|\Delta a_{i}\right\|^{p}\right)^{1 / p}\left(\sum_{i=1}^{n-1}\left\|\Delta b_{i}\right\|^{p}\right)^{1 / p} \\
& =\left[\frac{1}{4}(n+1)^{2}\right]^{\frac{q-1}{q}}\left(\sum_{i=1}^{n-1} \sum_{j=1}^{n-1}\left(\sum_{k=1}^{\min \{i, j\}}\left|p_{k}\right|^{q} \sum_{k=\max \{i, j\}}^{n}\left|p_{k}\right|^{q}\right)\right)^{1 / q} \\
& \times\left(\sum_{i=1}^{n-1}\left\|\Delta a_{i}\right\|^{p}\right)^{1 / p}\left(\sum_{i=1}^{n-1}\left\|\Delta b_{i}\right\|^{p}\right)^{1 / p} \\
& =\frac{1}{4^{p}}(n+1)^{2 p}\left(\sum_{i=1}^{n-1} \sum_{j=1}^{n-1}\left(\sum_{k=1}^{\min \{i, j\}}\left|p_{k}\right|^{q} \sum_{k=\max \{i, j\}}^{n}\left|p_{k}\right|^{q}\right)\right)^{1 / q} \\
& \times\left(\sum_{i=1}^{n-1}\left\|\Delta a_{i}\right\|^{p}\right)^{1 / p}\left(\sum_{i=1}^{n-1}\left\|\Delta b_{i}\right\|^{p}\right)^{1 / p} .
\end{aligned}
$$

If we use the identity for real numbers

$$
\mathrm{T}_{\mathrm{n}}(\mathbf{q} ; \mathbf{a}, \mathbf{b})=\sum_{i=1}^{\mathrm{n}-1} \sum_{j=1}^{\mathrm{n}-1} \mathrm{Q}_{\min \{i, j\}} \overline{\mathrm{Q}}_{\max \{i, j\}} \Delta \mathrm{a}_{j} \Delta \mathrm{b}_{i} .
$$

and the choices $a_{j}=b_{j}=j$ and $q_{k}=\left|p_{k}\right|^{q}$, then we get

$$
\sum_{i=1}^{n-1} \sum_{j=1}^{n-1}\left(\sum_{k=1}^{\min \{i, j\}}\left|p_{k}\right|^{q} \sum_{k=\max \{i, j\}}^{n}\left|p_{k}\right|^{q}\right)=\sum_{i=1}^{n}\left|p_{i}\right|^{q} \sum_{i=1}^{n} i^{2}\left|p_{i}\right|^{q}-\left(\sum_{i=1}^{n} i\left|p_{i}\right|^{q}\right)^{2} \text {. }
$$


Replacing this in (4.13) produces the desired result (4.11).

The following corollary also holds. It was obtained earlier in the paper with a different proof.

Corolary 5. Let $\mathbf{p}=\left(\mathrm{p}_{1}, \ldots, \mathrm{p}_{\mathrm{n}}\right) \in \mathbb{K}^{n}$, and $\boldsymbol{a}=\left(\mathrm{a}_{1}, \ldots, \mathrm{a}_{\mathrm{n}}\right), \boldsymbol{b}=\left(\mathrm{b}_{1}, \ldots, \mathrm{b}_{\mathrm{n}}\right) \in \mathcal{B}^{\mathrm{n}}$. Then we have the inequality

$$
\begin{aligned}
\left\|T_{n}(\mathbf{p} ; \mathbf{a}, \mathbf{b})\right\| & \leq\left[\sum_{i=1}^{n}\left|p_{i}\right| \sum_{i=1}^{n} i^{2}\left|p_{i}\right|-\left(\sum_{i=1}^{n} i\left|p_{i}\right|\right)^{2}\right] \\
& \times \max _{1 \leq i \leq n-1}\left\|\Delta a_{i}\right\| \max _{1 \leq i \leq n-1}\left\|\Delta b_{i}\right\| .
\end{aligned}
$$

The inequality is sharp.

Proof. From the third inequality in (4.8) we have

$$
\begin{aligned}
& \left\|T_{n}(\mathbf{p} ; \mathbf{a}, \mathbf{b})\right\| \\
& \leq \max _{1 \leq i \leq n-1}\left\|\Delta a_{i}\right\| \max _{1 \leq i \leq n-1}\left\|\Delta b_{i}\right\| \sum_{i=1}^{n-1} \sum_{j=1}^{n-1}\left|P_{\min \{i, j\}}\right|\left|\bar{P}_{\max \{i, j\}}\right| \\
& =\max _{1 \leq i \leq n-1}\left\|\Delta a_{i}\right\| \max _{1 \leq i \leq n-1}\left\|\Delta b_{i}\right\| \sum_{i=1}^{n-1} \sum_{j=1}^{n-1}\left|\sum_{k=1}^{\min \{i, j\}} p_{k}\right|\left|\sum_{k=\max \{i, j\}}^{n} p_{k}\right| \\
& \leq \max _{1 \leq i \leq n-1}\left\|\Delta a_{i}\right\| \max _{1 \leq i \leq n-1}\left\|\Delta b_{i}\right\| \sum_{i=1}^{n-1} \sum_{j=1}^{n-1}\left(\sum_{k=1}^{\min \{i, j\}}\left|p_{k}\right| \sum_{k=\max \{i, j\}}^{n}\left|p_{k}\right|\right) \\
& =\left[\sum_{i=1}^{n}\left|p_{i}\right| \sum_{i=1}^{n} i^{2}\left|p_{i}\right|-\left(\sum_{i=1}^{n} i\left|p_{i}\right|\right)^{2}\right]
\end{aligned}
$$

and the desired inequality (4.14) is proved.

The sharpness of the inequality follows as above and the details are omitted.

\section{Inequalities for Power Series}

We have:

Theorem 5.1. Let $f(\lambda)=\sum_{n=0}^{\infty} \alpha_{n} \lambda^{n}$ be a power series that is convergent on the open disk $\mathrm{D}(0, \mathrm{R})$, with $\mathrm{R}>0$. If $\mathrm{x}, \mathrm{y} \in \mathcal{B}$ with $\mathrm{xy}=\mathrm{yx}$ and $\|\mathrm{x}\|,\|\mathrm{y}\|<1$, then we have for $\lambda \in \mathbb{C}$ with $|\lambda|<\mathrm{R}$ the inequality:

$$
\|\tilde{f}(\lambda \cdot 1) \tilde{f}(\lambda x y)-\tilde{f}(\lambda x) \tilde{f}(\lambda y)\| \leq \frac{1}{4} \cdot \frac{\|x-1\|\|y-1\|}{(1-\|x\|)(1-\|y\|)} f_{A}^{2}(|\lambda|) .
$$


Proof. Utilising the inequality (4.9) we have for all $n \geq 1$

$$
\begin{aligned}
& \left\|\sum_{i=0}^{n} \alpha_{i} \lambda^{i} \sum_{i=0}^{n} \alpha_{i} \lambda^{i} x^{i} y^{i}-\sum_{i=0}^{n} \alpha_{i} \lambda^{i} x^{i} \sum_{i=0}^{n} \alpha_{i} \lambda^{i} y^{i}\right\| \\
& \leq \frac{1}{4}\left(\sum_{k=0}^{n}\left|\alpha_{k}\right||\lambda|^{k}\right)^{2} \sum_{i=0}^{n-1}\left\|x^{i+1}-x^{i}\right\| \sum_{i=0}^{n-1}\left\|y^{i+1}-y^{i}\right\| .
\end{aligned}
$$

Observe that

$$
\begin{aligned}
\sum_{i=0}^{n-1}\left\|x^{i+1}-x^{i}\right\| & =\sum_{i=0}^{n-1}\left\|x^{i}(x-1)\right\| \leq\|x-1\| \sum_{i=0}^{n-1}\left\|x^{i}\right\| \\
& \leq\|x-1\| \sum_{i=0}^{n-1}\|x\|^{i}=\|x-1\| \frac{1-\|x\|^{n}}{1-\|x\|}
\end{aligned}
$$

and, similarly,

$$
\sum_{i=0}^{n-1}\left\|y^{i+1}-y^{i}\right\| \leq\|y-1\| \frac{1-\|y\|^{n}}{1-\|y\|}
$$

Utilizing (5.2) and the fact that $x y=y x$, we have

$$
\begin{aligned}
& \left\|\sum_{i=0}^{n} \alpha_{i} \lambda^{i} \sum_{i=0}^{n} \alpha_{i} \lambda^{i}(x y)^{i}-\sum_{i=0}^{n} \alpha_{i} \lambda^{i} x^{i} \sum_{i=0}^{n} \alpha_{i} \lambda^{i} y^{i}\right\| \\
& \leq \frac{1}{4}\|x-1\|\|y-1\|\left(\sum_{k=0}^{n}\left|\alpha_{k}\right||\lambda|^{k}\right)^{2} \frac{1-\|x\|^{n}}{1-\|x\|} \frac{1-\|y\|^{n}}{1-\|y\|}
\end{aligned}
$$

for any $n \geq 1$.

Since all the series whose partial sums are involved in (5.3) are convergent, then by letting $\mathrm{n} \rightarrow \infty$ in (5.3) we deduce the desired inequality (5.1).

Corolary 6. Let $f(\lambda)=\sum_{n=0}^{\infty} \alpha_{n} \lambda^{n}$ be a power series that is convergent on the open disk $D(0, R)$, with $\mathrm{R}>0$. If $x \in \mathcal{B}$ and $\|x\|<1$, then we have for $\lambda \in \mathbb{C}$ with $|\lambda|<\mathrm{R}$ the inequality:

$$
\left\|\tilde{f}(\lambda \cdot 1) \tilde{f}\left(\lambda x^{2}\right)-[\widetilde{f}(\lambda x)]^{2}\right\| \leq \frac{1}{4} \cdot \frac{\|x-1\|^{2}}{\left(1-\|x\|^{2}\right.} f_{A}^{2}(|\lambda|) .
$$


As some natural examples that are useful for applications, we can point out that, if

$$
\begin{aligned}
& f(\lambda)=\sum_{n=1}^{\infty} \frac{(-1)^{n}}{n} \lambda^{n}=\ln \frac{1}{1+\lambda}, \lambda \in D(0,1) ; \\
& g(\lambda)=\sum_{n=0}^{\infty} \frac{(-1)^{n}}{(2 n) !} \lambda^{2 n}=\cos \lambda, \lambda \in \mathbb{C} ; \\
& h(\lambda)=\sum_{n=0}^{\infty} \frac{(-1)^{n}}{(2 n+1) !} \lambda^{2 n+1}=\sin \lambda, \lambda \in \mathbb{C} ; \\
& l(\lambda)=\sum_{n=0}^{\infty}(-1)^{n} \lambda^{n}=\frac{1}{1+\lambda}, \lambda \in D(0,1) ;
\end{aligned}
$$

then the corresponding functions constructed by the use of the absolute values of the coefficients are

$$
\begin{aligned}
& \mathrm{f}_{\mathrm{A}}(\lambda)=\sum_{n=1}^{\infty} \frac{1}{n} \lambda^{n}=\ln \frac{1}{1-\lambda}, \lambda \in \mathrm{D}(0,1) ; \\
& \mathrm{g}_{\mathrm{A}}(\lambda)=\sum_{n=0}^{\infty} \frac{1}{(2 n) !} \lambda^{2 n}=\cosh \lambda, \lambda \in \mathbb{C} ; \\
& \mathrm{h}_{\mathrm{A}}(\lambda)=\sum_{n=0}^{\infty} \frac{1}{(2 n+1) !} \lambda^{2 n+1}=\sinh \lambda, \lambda \in \mathbb{C} ; \\
& \mathrm{l}_{\mathrm{A}}(\lambda)=\sum_{n=0}^{\infty} \lambda^{n}=\frac{1}{1-\lambda}, \lambda \in \mathrm{D}(0,1) .
\end{aligned}
$$

Other important examples of functions as power series representations with nonnegative coefficients are:

$$
\begin{aligned}
\exp (\lambda) & =\sum_{n=0}^{\infty} \frac{1}{n !} \lambda^{n} \quad \lambda \in \mathbb{C} \\
\frac{1}{2} \ln \left(\frac{1+\lambda}{1-\lambda}\right) & =\sum_{n=1}^{\infty} \frac{1}{2 n-1} \lambda^{2 n-1}, \quad \lambda \in D(0,1) ; \\
\sin ^{-1}(\lambda) & =\sum_{n=0}^{\infty} \frac{\Gamma\left(n+\frac{1}{2}\right)}{\sqrt{\pi}(2 n+1) n !} \lambda^{2 n+1}, \quad \lambda \in D(0,1) ; \\
\tanh ^{-1}(\lambda) & =\sum_{n=1}^{\infty} \frac{1}{2 n-1} \lambda^{2 n-1}, \quad \lambda \in D(0,1) \\
{ }_{2} F_{1}(\alpha, \beta, \gamma, \lambda) & =\sum_{n=0}^{\infty} \frac{\Gamma(n+\alpha) \Gamma(n+\beta) \Gamma(\gamma)}{n ! \Gamma(\alpha) \Gamma(\beta) \Gamma(n+\gamma)} \lambda^{n}, \alpha, \beta, \gamma>0 \\
\lambda & \in D(0,1) ;
\end{aligned}
$$

where $\Gamma$ is Gamma function. 
Example 1. a) If $x, y \in \mathcal{B}$ with $x y=y x$ and $\|x\|,\|y\|<1$, then we have for $\lambda \in \mathbb{C}$ the inequality:

$$
\|\exp [\lambda(1+x y)]-\exp [\lambda(x+y)]\| \leq \frac{1}{4} \cdot \frac{\|x-1\|\|y-1\|}{(1-\|x\|)(1-\|y\|)} \exp (2|\lambda|) .
$$

In particular, we have

$$
\left\|\exp \left[\lambda\left(1+x^{2}\right)\right]-\exp [2 \lambda x]\right\| \leq \frac{1}{4} \cdot \frac{\|x-1\|^{2}}{(1-\|x\|)^{2}} \exp (2|\lambda|)
$$

and

$$
\left\|\exp \left[\lambda\left(1-\chi^{2}\right)\right]-1\right\| \leq \frac{1}{4} \cdot \frac{\|x-1\|\|x+1\|}{(1-\|x\|)^{2}} \exp (2|\lambda|)
$$

for any $x \in \mathcal{B}$ with $\|x\|<1$ and $\lambda \in \mathbb{C}$.

b) We have the inequality

$$
\begin{aligned}
& \left\|(1-\lambda)^{-1}(1-\lambda x y)^{-1}-(1-\lambda x)^{-1}(1-\lambda y)^{-1}\right\| \\
& \leq \frac{1}{4} \cdot \frac{\|x-1\|\|y-1\|}{(1-\|x\|)(1-\|y\|)(1-|\lambda|)^{2}}
\end{aligned}
$$

for any $\mathrm{x}, \mathrm{y} \in \mathcal{B}$ with $\mathrm{xy}=\mathrm{yx},\|\mathrm{x}\|,\|\mathrm{y}\|<1$ and $\lambda \in \mathbb{C}$ with $|\lambda|<1$.

In particular, we have

$$
\left\|(1-\lambda)^{-1}\left(1-\lambda x^{2}\right)^{-1}-(1-\lambda x)^{-2}\right\| \leq \frac{1}{4} \cdot \frac{\|x-1\|^{2}}{(1-\|x\|)^{2}(1-|\lambda|)^{2}}
$$

and

$$
\begin{aligned}
& \left\|(1-\lambda)^{-1}\left(1+\lambda x^{2}\right)^{-1}-(1-\lambda x)^{-1}(1+\lambda x)^{-1}\right\| \\
& \leq \frac{1}{4} \cdot \frac{\|x-1\|\|x+1\|}{(1-\|x\|)^{2}(1-|\lambda|)^{2}}
\end{aligned}
$$

for any $x \in \mathcal{B}$ with $\|x\|<1$ and $\lambda \in \mathbb{C}$ with $|\lambda|<1$.

c) We have the inequality

$$
\begin{aligned}
& \left\|\ln (1-\lambda)^{-1} \ln (1-\lambda x y)^{-1}-\ln (1-\lambda x)^{-1} \ln (1-\lambda y)^{-1}\right\| \\
& \leq \frac{1}{4} \cdot \frac{\|x-1\|\|y-1\|}{(1-\|x\|)(1-\|y\|)}[\ln (1-|\lambda|)]^{2}
\end{aligned}
$$

for any $\mathrm{x}, \mathrm{y} \in \mathcal{B}$ with $\mathrm{xy}=\mathrm{yx},\|\mathrm{x}\|,\|\mathrm{y}\|<1$ and $\lambda \in \mathbb{C}$ with $|\lambda|<1$.

In particular, we have

$$
\left\|(1-\lambda)^{-1}\left(1-\lambda x^{2}\right)^{-1}-(1-\lambda x)^{-2}\right\| \leq \frac{1}{4} \cdot \frac{\|x-1\|^{2}}{(1-\|x\|)^{2}}[\ln (1-|\lambda|)]^{2}
$$


and

$$
\begin{aligned}
& \left\|(1-\lambda)^{-1}\left(1+\lambda x^{2}\right)^{-1}-(1-\lambda x)^{-1}(1+\lambda x)^{-1}\right\| \\
& \leq \frac{1}{4} \cdot \frac{\|x-1\|\|x+1\|}{(1-\|x\|)^{2}}[\ln (1-|\lambda|)]^{2}
\end{aligned}
$$

for any $x \in \mathcal{B}$ with $\|x\|<1$ and $\lambda \in \mathbb{C}$ with $|\lambda|<1$.

Acknowledgement. The authors would like to thank the anonymous referee for valuable suggestions that have been implemented in the final version of the paper.

\section{References}

[1] G. A. Anastassiou, Grüss type inequalities for the Stieltjes integral. Nonlinear Funct. Anal. Appl. 12 (2007), no. 4, 583-593.

[2] M. Biernacki, Sur une inégalité entre les intégrales due à Tchebyscheff. Ann. Univ. Mariae Curie-Sklodowska (Poland), A5(1951), 23-29.

[3] M. V. Boldea, S. S. Dragomir and M. Megan, New bounds for Chebyshev functional for power series in Banach algebras via a Grüss-Lupaş type inequality, PanAmerican Mathematical Journal, 26(2016), 71 - 88.

[4] K. Boukerrioua, and A. Guezane-Lakoud, On generalization of Chebyshev type inequalities. J. Inequal. Pure Appl. Math. 8 (2007), no. 2, Article 55, 4 pp.

[5] P. L. Čebyšev, O približennyh vyraženijah odnih integralov čerez drugie. Soobšćenija i protokoly zasedaniŭ Matemmatičeskogo občestva pri Imperatorskom Har'kovskom Universitete No. 2, 93-98; Polnoe sobranie sočinenǐ P. L. Čebyševa. Moskva-Leningrad, 1948a, (1882), 128131.

[6] P.L. Čebyšev, Ob odnom rjade, dostavljajušćem predel'nye veličiny integralov pri razloženii podintegral'noĭ funkcii na množeteli. Priloženi k 57 tomu Zapisok Imp. Akad. Nauk, No. 4; Polnoe sobranie sočineniu P. L. Čebyševa. Moskva-Leningrad, 1948b, (1883),157-169.

[7] P. Cerone, On a Chebyshev-type functional and Grüss-like bounds. Math. Inequal. Appl. 9 (2006), no. 1, 87-102.

[8] P. Cerone and S. S. Dragomir, A refinement of the Grüss inequality and applications. Tamkang J. Math. 38 (2007), no. 1, 37-49.

[9] P. Cerone and S. S. Dragomir, Chebychev functional bounds using Ostrowski seminorms. Southeast Asian Bull. Math. 28 (2004), no. 2, 219-228. 
[10] P. Cerone and S. S. Dragomir, New bounds for the Čebyšev functional. Appl. Math. Lett. 18 (2005), no. 6, 603-611.

[11] S. S. Dragomir, Inequalities for the Chebyshev functional of two functions of selfadjoint operators in Hilbert spaces , Aust. J. Math. Anal. \& Appl. 6(2009), Issue 1, Article 7, pp. $1-58$.

[12] S. S. Dragomir, Some inequalities for power series of selfadjoint operators in Hilbert spaces via reverses of the Schwarz inequality. Integral Transforms Spec. Funct. 20 (2009), no. 9-10, $757-767$.

[13] S.S. Dragomir, A generalization of Grüss' inequality in inner product spaces and applications, J. Math. Anal. Appl., 237 (1999), 74-82.

[14] S.S. Dragomir, Some integral inequalities of Grüss type, Indian J. of Pure and Appl. Math., 31(4) (2000), 397-415.

[15] S. S. Dragomir, Some Grüss type inequalities in inner product spaces, J. Inequal. Pure 63 Appl. Math., 4(2) (2003), Article 42. (Online http://jipam.vu.edu.au/article.php?sid=280).

[16] S. S. Dragomir, Reverses of Schwarz, triangle and Bessel inequalities in inner product spaces, J. Inequal. Pure \&3 Appl. Math., 5(3) (2004), Article 76. (Online : http://jipam.vu.edu.au/article.php?sid=432).

[17] S.S. Dragomir, New reverses of Schwarz, triangle and Bessel inequalities in inner product spaces, Austral. J. Math. Anal. \& Applics., 1(1) (2004), Article 1. (Online: http://ajmaa.org/cgi-bin/paper.pl?string=nrstbiips.tex $)$.

[18] S. S. Dragomir, On the Chebyshev's inequality for weighted means. Acta Math. Hungar. 104 (2004), no. 4, 345-355.

[19] S.S. Dragomir, On Bessel and Grüss inequalities for orthornormal families in inner product spaces, Bull. Austral. Math. Soc., 69(2) (2004), 327-340.

[20] S. S. Dragomir, Advances in Inequalities of the Schwarz, Grüss and Bessel Type in Inner Product Spaces, Nova Science Publishers Inc, New York, 2005, x+249 p.

[21] S. S. Dragomir, Reverses of the Schwarz inequality in inner product spaces generalising a Klamkin-McLenaghan result, Bull. Austral. Math. Soc. 73(1)(2006), 69-78.

[22] S. S. Dragomir, Operator Inequalities of the Jensen, Chebyshev and Grüss Type. Springer Briefs in Mathematics. Springer, New York, 2012. xii+121 pp. ISBN: 978-1-4614-1520-6.

[23] S. S. Dragomir, Operator Inequalities of Ostrowski and Trapezoidal Type. Springer Briefs in Mathematics. Springer, New York, 2012. x+112 pp. ISBN: 978-1-4614-1778-1. 
[24] S. S. Dragomir, M. V. Boldea, C. Buşe and Mihail Megan, Norm inequalities of Chebyshev type for power series in Banach algebras, J. Inequal. Appl., 2014, 2014:294.

[25] S. S. Dragomir and G.L. Booth, On a Grüss-Lupaş type inequality and its applications for the estimation of p-moments of guessing mappings, Mathematical Communications, 5(2000), $117-126$.

[26] S. S. Dragomir and I. Fedotov, An inequality of Grüss' type for Riemann-Stieltjes integral and applications for special means, Tamkang J. of Math., 29(4)(1998), 286-292.

[27] S. S. Dragomir and B. Mond, Some mappings associated with Čebyšev's inequality for sequences of real numbers. Bull. Allahabad Math. Soc. $8 / 9$ (1993/94), 37-55.

[28] S.S. Dragomir and J.E. Pečarić, Refinements of some inequalities for isotonic linear functionals, L'Anal. Num. Théor de L'Approx. (Romania) 18(1989) (1), 61-65.

[29] S. S. Dragomir, J. Pečarić and J. Sándor, The Chebyshev inequality in pre-Hilbertian spaces. II. Proceedings of the Third Symposium of Mathematics and its Applications (Timisoara, 1989), 75-78, Rom. Acad., Timişoara, 1990. MR1266442 (94m:46033)

[30] S. S. Dragomir and J. Sándor, The Chebyshev inequality in pre-Hilbertian spaces. I. Proceedings of the Second Symposium of Mathematics and its Applications (Timisoara, 1987), 61-64, Res. Centre, Acad. SR Romania, Timişoara, 1988. MR1006000 (90k:46048).

[31] A. M. Fink, A treatise on Grüss' inequality, Analytic and Geometric Inequalities, 93-113, Math. Appl. 478, Kluwer Academic Publ., 1999.

[32] T. Furuta, J. Mićić Hot, J. Pečarić and Y. Seo, Mond-Pečarić Method in Operator Inequalities. Inequalities for Bounded Selfadjoint Operators on a Hilbert Space, Element, Zagreb, 2005.

[33] G. Grüss, Über das Maximum des absoluten Betrages von $\frac{1}{b-a} \int_{a}^{b} f(x) g(x) d x-$ $\frac{1}{(b-a)^{2}} \int_{a}^{b} f(x) d x \int_{a}^{b} g(x) d x$, Math. Z., 39(1935), 215-226.

[34] G. H. Hardy, J. E. Littlewood and G. Pólya, Inequalities, 1st Ed. and 2nd Ed. Cambridge University Press, $(1934,1952)$ Cambridge, England.

[35] Z. Liu, Refinement of an inequality of Grüss type for Riemann-Stieltjes integral, Soochow J. Math., 30(4) (2004), 483-489.

[36] A. Matković, J. Pečarić and I. Perić, A variant of Jensen's inequality of Mercer's type for operators with applications. Linear Algebra Appl. 418 (2006), no. 2-3, 551-564.

[37] D. S. Mitrinović and J.E. Pečarić, History, variations and generalizations of the Chebyshev inequality and question of some priorities. II. Rad Jugoslav. Akad. Znan. Umjet. No. 450 (1990), 139-156. 
[38] D. S. Mitrinović and J.E. Pečarić, On an identity of D.Z. Djoković, Prilozi Mak. Akad.Nauk. Umj. (Skopje), 12(1)(1991), 21-22.

[39] D. S. Mitrinović, J. E. Pečarić and A. M. Fink, Classical and New Inequalities in Analysis, Kluwer Academic Publishers, Dordrecht, 1993.

[40] D. S. Mitrinović and P.M. Vasić, History, variations and generalisations of the Chebyshev inequality and the question of some priorities. Univ. Beograd. Publ. Elektrotehn. Fak. Ser. Mat. Fiz. No. 461-497 (1974), 1-30.

[41] J. Pečarić, Remarks on Biernacki's generalization of Chebyshev's inequality. Ann. Univ. Mariae Curie-Sklodowska Sect. A 47 (1993), 116-122.

[42] J. E. Pečarić and S.S. Dragomir, Some remarks on Chebyshev's inequality, L'Anal. Num. Théor de L'Approx. 19 (1)(1990), 58-65.

[43] J. Pečarić, J. Mićić and Y. Seo, Inequalities between operator means based on the MondPečarić method. Houston J. Math. 30 (2004), no. 1, 191-207

[44] C.-J. Zhao and W.-S. Cheung, On multivariate Grüss inequalities. J. Inequal. Appl. 2008, Art. ID 249438,8 pp. 